\title{
Mustakabali wa Kiswahili kama Lugha ya Kufundishia Nchini Tanzania: Uhakiki wa Maudhui ya Sera ya Elimu na Mafunzo ya 2014
}

\author{
Gervas A. Kawonga ${ }^{1}$
}

\begin{abstract}
Ikisiri
Msingi wa makala haya ni "Sera ya Elimu na Mafunzo" (SEM, 2014). Makala yanahakiki nafasi ya lugha ya Kiswahili kwenye Sera ya Elimu na Mafunzo nchini Tanzania na mustakabali wake kwenye mfumo wa elimu kama lugha ya kufundishia. Mbinu iliyotumika ni ya maktabani ambapo mwandishi alisoma na kuhakiki maudhui ya SEM msisitizo ukiwa kwenye masuala yahusuyo lugha ya kufundishia. Uchambuzi wa kiisimujamii ulitumika ili kuonesha ukinzani baina ya matamko ya SEM. Tamko la SEM kuhusu lugha ya Kiswahili kama lugha ya kufundishia haliakisi hali halisi. Uhusiano wa Kiisimujamii wa lugha ya Kiswahili na ya Kiingereza kuhusu elimu unatoa tafsiri kuwa endapo SEM itatumika bila nia ya dhati ya kuisaidia lugha ya Kiswahili basi lugha hiyo inaweza kutoweka kabisa kwenye mfumo wa elimu kama lugha ya kufundishia. Makala yanajenga hoja kuwa tamko la SEM kwamba Kiingereza na Kiswahili ziwe na hadhi sawa kwenye mfumo wa elimu linatia moyo kwa mustakabali wa lugha ya Kiswahili lakini utekelezaji wake unaibua changamoto nyingi kwa namna ambayo zisipotazamwa kwa makini lugha ya Kiingereza itaendelea kukita mizizi zaidi na kujenga uwezekano wa jamii kukataa kufundishwa kwa lugha ya Kiswahili kwa sababu mwelekeo wa jamii unanasibisha elimu na Kiingereza. Mwisho, wito unatolewa kwa watunga sera kusitisha kutumia lugha ya wachache kutoa elimu kwa wengi kwa kuwa kufanya hivyo ni kubadili malengo ya elimu.
\end{abstract}

Istilahi Muhimu: sera ya elimu; sera ya lugha; lugha ya kufundishia; Tanzania DOI: https://doi.org/10.37759/mjed.2017.1.1.6

\subsection{Utangulizi}

Lugha mama ina athari kubwa katika fikra za mtu kuhusu ulimwengu wake, imani, falsafa na mtazamo juu ya ulimwengu. Kila binadamu hujiundia ulimwengu wake ambao ni faragha isiyo wezi kufanana na ulimwengu wa mtu mwingine. Mtu anayetaka kuujua ulimwengu wa mtu mwingine lazima afanye juhudi za kuujua na anaweza kufanikiwa kwa kiasi kidogo tu kupitia lugha ya mtu anayehusika (Rogers, 1969; Boroditsky, 2001). Temples (1959) alidai kuwa ukitaka kujua falsafa ya watu duni, sharti ujue lugha yao. Madai haya ya kisaikolojia na kifalsafa yanaelekea kutozingatiwa katika sera za lugha katika nchi nyingi za Afrika, Tanzania ikiwa mojawapo. Hali hiyo inatokea licha ya ukweli kwamba lugha ndiyo nyenzo ya kufikiri na maarifa.

Mjadala juu ya lugha gani itumike kufundishia nchini Tanzainia umedumu kwa zaidi ya miongo mitatu sasa tofauti na madai ya awali kwamba umedumu kwa miongo miwili. Tanzania ina lugha za asili zipatazo 150 zilizosambaa nchi nzima, Kiswahili kikiwa mojawapo (Tibategeza, 2009). Vilevile, kuna lugha za kigeni kama Kiingereza, Kifaransa,

\footnotetext{
${ }^{1}$ Chuo Kikuu Kishiriki cha Elimu Mkwawa; Baruapepe: kawongag@ yahoo.com
} 
Kiarabu na Kichina. Hata hivyo, ni Kiingereza na Kiswahili tu ndizo lugha ambazo zimepewa hadhi rasmi. Kiswahili ni lugha ya taifa, lugha ya kufundishia elimu ya awali na msingi na mafunzo ya ualimu ngazi ya cheti na lugha ya uendeshaji wa shughuli za serikali ikiwa ni pamoja na mashauri katika mahakama za mwanzo. Kiingereza ni lugha ya uendeshaji wa shughuli za serikali, diplomasia, mahakama kuu na mahakama za hakimu mkazi pamoja na mahakama za rufaa. Pia Kiingereza ni lugha ya kufundishia elimu ya sekondari na elimu ya juu. Mgawanyo huu wa dhima kama utakavyoelezwa badaye, kiisimujamii haujakitwa kwenye hali ya usawa. Ni kutokana na hali hiyo uzoefu wa Watanzania wengi kuhusu wanavyoyafahamu mazingira yao unaathiriwa na lugha hizo mbili, yaani Kiingereza na Kiswahili, kwa mielekeo na viwango vinavyotofautiana.

Tafiti mbalimbali (BAKITA, 2004; Malekela, 2006; Rutalemwa, 2009) wanakubaliana kwamba zaidi ya $90 \%$ ya Watanzania wanazungumza Kiswahili ama kama lugha ya kwanza au kama lugha ya pili. Kutokana na umilisi wa kiwango cha juu unaofikiwa na watumiaji wengi wa lugha ya Kiswahili baadhi ya tafiti (Mekacha, 1994; Rubanza, 1996) zina madai kwamba watoto wa Kitanzania huamilia lugha ya Kiswahili kwa pamoja na lugha zao za asili. Madai hayo yanatokana na ukweli kwambamsingi wa kuamilia lugha ni muwasala wa kijamii wa lugha husika (Krashen, 1981). Mazingira ya Tanzania yanawafanya watoto kuwa na muwasala wa kijamii wa lugha ya Kiswahili na lugha zao za asili hali inayowawezesha kuamilia Kiswahili kwa pamoja na lugha zao za asili. Kutokana na uamiliaji huo wa pamoja, wamilisi wengi wa lugha ya Kiswahili hushindwa kuweka mpaka ulio wazi kwamba ni lini walimaliza kujifunza lugha ya asili na lini walianza kujifunza Kiswahili. Hata hivyo, watu wengi huchukulia kwa mazoea kwamba watoto wanajifunza Kiswahili wanapoanza elimu ya msingi huku wakisahau kuwa kuna ambao wanakosa fursa ya kwenda shule lakini wanamudu lugha yao ya asili na Kiswahili kwa viwango visivyotofautiana. Kwa hiyo, urazini wa Watanzania na uzoefu kuhusu mazingira yao umefinyangwa zaidi na lugha ya Kiswahili na lugha za asili kuliko lugha za kigeni kama ilivyo Kiingereza. Hii ni kwa sababu lugha ambayo mtu anaifahamu vyema ndiyo inayoathiri zaidi jinsi anavyo ufahamu ulimwengu na kuufasiri.

\section{Mbinu za Utafiti}

Mbinu iliyotumika ni ya utafiti wa maktabani. Mtafiti alisoma maandiko kuhusu Sera ya Elimu na Mafunzo, 2014 (SEM, 2014) msisitizo ukiwa jinsi suala la lugha ya kufundishia linavyozingatiwa. SEM, 2014) ndiyo msingi wa makala haya ambayo pamoja na mambo mengine inaweka bayana lugha ipi iwe ya kufundishia katika ngazi mbalimbali za elimu. Uteuzi wa matini hii ulitokana na ukweli kwamba mjadala wa lugha kisera una takribani miongo mitatu na katika nyakati tofauti lugha ya Kiswahili imekuwa ikipewa dhima za chini ikilinganishwa na lugha ya Kiingereza. Tofauti na hali hiyo ya awali katika SEM, 2014 lugha ya Kiswahili na lugha ya Kiingereza zinapewa nafasi sawa kwenye mfumo wa elimu kama lugha za kufundishia. Kwa maneno mengine kunahitajika lugha uwili kwenye mfumo wa elimu. Mwandishi alihakiki kama maudhui ya SEM, 2014 kuhusu lugha ya kufundishia yanatoa nafasi ya kuimarika au kudhohofisha lugha ya Kiswahii kwenye mfumo wa elimu. Maudhui ya SEM, 2014 yalihakikiwa ili kubainisha kama kuna mustakabali wa Kiswahili kama lugha ya kufundishia au la.

\section{Mkabala wa Kinadharia}


Makala haya yanaongozwa na mkabala wa kinadharia wa kiisimujamiii ujulikanao kama Mgongano wa Daiglosia ambao uliasisiwa na Fasold (1990). Msingi mkuu wa mtazamo huu ni kuwa jamiilugha yoyote huwa imegawanyika katika matabaka yanayotofautiana katika nguvu za kiuchumi, kisiasa na kijamii. Tofauti hizo zinajidhihirisha pia katika mgawanyiko wa nafasi, dhima na uamilifu wa lugha katika jamii husika. Kwa mujibu wa Mekacha (2011), lugha inayofungamana na tabaka lenye nguvu inakuwa na nafasi ya juu kiuamilifu kuliko ile inayofungamana na tabaka duni. Nchini Tanzania, kwa mfano, Kiingereza kina hadhi ya juu zaidi kuliko Kiswahili. Vilevile ingawa Kiswahili ni miongoni mwa lugha za asili lakini kihadhi lugha ya Kiswahili ina hadhi ya juu kuliko lugha zingine za asili. Tofauti ya hadhi za lugha hizo kwa mujibu wa nadharia hii ni tofauti za matabaka ya jamiilugha husika.

Katika muktadha wa ki-daiglosia, mgongano hutokea baina ya lugha yenye hadhi ya juu na lugha zenye hadhi ya chini zinazowania hadhi ya juu ikiwa ni pamoja na nafasi ya kuwa lugha ya kufundishia. Mgongano huo wa lugha kimsingi ni mgongano baina ya matabaka ya watumiaji wa lugha tofauti kwa sababu mafanikio katika ushindani wa lugha ni kigezo kimojawapo kikubwa kinachoukilia ushindi wa kisiasa, kiuchumi na kiutamaduni. Nadharia ya Mgongano wa daiglosia inatoa nafasi ya kuhakiki jinsi lugha mbalimbali katika jamiilughaulumbi, kama ilivyo Tanzania, zinavyopewa dhima kupitia mamlaka za maamuzi. SEM, 2014, kwa mkabala huu wa kinadharia, na kwa kuzingatia madai ya Mekacha (1995) kuhusu sera, ni mbinu ya kuhalalisha taratibu za tabaka lenye nguvu kulinda maslahi yake.

Kwa hiyo, katika muktadha wa makala haya SEM, 2014 ni mbinu ya kuhalalisha Kiingereza kiwe lugha ya kufundishia katika ngazi zote za elimu hata kama hakina tija kwa wanyonge walio wengi. Nadharia hii inasaidia kuonesha kuwa maudhui ya SEM, 2014 kuhusu mpango wa lugha ni matokeo ya mivutano ya kitabaka. Kwa mfano, wakati kwa upande mmoja SEM, 2014 inaonesha matumaini makubwa kwa lugha ya Kiswahili ni SEM, 2014 hiyohiyo inaziweka lehani lugha zingine za asili ukiacha Kiswahili. Hata hivyo, nafasi ya lugha zingine za asili tofauti na Kiswahili katika SEM, 2014 litahitaji mjadala wa peke yake. Jinsi lugha hizo zinavyohusishwa au kuachwa kwenye sera si bahati mbaya bali ni jitihada zenye utashi wa kuliridhisha matabaka tofauti yenye maslahi kwenye lugha tofauti. SEM, 2014 inazipa Kiswahili na Kiingereza nafasi sawa ambayo kiuhalisia inazua changamoto za kiutekelezaji. Kusudio la kuzipa lugha hizo hadhi sawa ni kutoa matumaini kwa tabaka la watumiaji wa Kiswahili na kulifurahisha tabaka la juu la watumiaji wa lugha ya Kiingereza, tabaka la wachache ambao kiu yao ni kuona maarifa na ukwasi vinanasibishwa na Kiingereza katika jamii. Mambo hayo yanaakisi kile kilichoelezwa na Rubagumya (1989) kwamba migawanyiko baina ya wazungumzaji wa lugha inaakisi migawanyiko ya kitabaka inayoonesha jinsi nguvu za kiuchumi na kisiasa zinavyotumika.

Nafasi ya nadharia hii katika makala haya ni kutanabaisha kwamba suala la lugha gani iwe ya kufundishia ni suala la harakati za kitabaka za kudai haki ya kufundishwa kwa lugha inayoeleweka vizuri. Mochiwa (1996) anasisitiza kuwepo kwa harakati za kudai Kiswahili kiwe lugha ya kufundishia na sio kutoa mapendekezo kwa tabaka lenye nguvu linaloishia kuhalalisha maamuzi yenye maslahi kwake kwa kutumia sera kama kigezo cha urasmi na usorasmi. Kwamba Kiswahili na Kiingereza zimepewa hadhi sawa kwenye SEM, 2014 ni ukweli ulioandikwa ambao hadi sasa ni miaka mitatu bila mikakati ya utekelezaji wake. Hakuna jithada za kufuta kasumba kwamba elimu bora inapatikana kwa lugha ya 
Kiingereza pekee na kuunda sura mpya kwamba Kiswahili na Kiingereza sasa zote zinaweza kutoa elimu yenye tija, tena katika muktadha wa Tanzania lugha ya Kiswahili inaweza kutoa elimu bora zaidi kuliko Kiingereza. Katika hatua hii kuna haja ya kuangalia suala la lugha kisera tangu 1961 hadi sasa ili kuimarisha usuli wa mjadala wetu. Kipindi hiki kimeteuliwa kwa kuzingatia kwamba Tanganyika ilikuwa imepata uhuru na ilikuwa na "maamuzi yake" kisera. Hata hivyo, maamuzi huru ya kisera si jambo rahisi katika nchi masikini.

\section{Suala la Lugha Kisera 1961 - Hadi Sasa}

Kwa mujibu wa Tibategeza (2009) kuna zana mbili muhimu za serikali kuhusiana na sera ya lugha. Kwanza ni Sera ya Utamaduni ya 1985 (kwa hiyo, SU) na Sera ya Elimu na Mafunzo ya mwaka 1995 (kwahiyo, SEM, 1995). Kabla ya sera hizo hakukuwepo na sera, bali yalikuwepo matamko mbalimbali ya serikali ambayo kwa namna moja au nyingine yalisaidia kukuza lugha ya Kiswahili (Mulokozi, 2002, Mekacha, 2011). Baadhi ya matamko yaliyowahi kutolewa na kufanya kazi kama sera ya lugha isiyo rasmi ni kama ifuatavyo:

i. Kiswahili kutangazwa kuwa lugha ya taifa mnamo mwaka 1962.

ii. Kiswahili kutangazwa kuwa lugha ya kufundishia katika miaka saba ya elimu ya msingi na katika elimu ya ualimu daraja la tatu 1967.

iii. Sera ya Ujamaa na Kujitegemea. Hii ilikusanya watu wenye usuli tofauti wa kiisimu hivyo Kiswahili kikachukua nafasi ya kuwa lugha ya upatanisho 1967.

iv. Kiswahili kutangazwa kuwa lugha rasmi mnamo mwaka 1967.

v. Kupitishwa kwa Sera ya Elimu ya Kujitegemea 1968.

vi. Kuondolewa kwa cheti cha mtihani wa Cambridge 1970. Hii ililazimisha wanafunzi kujifunza na kufaulu Kiswahili ili waweze kupata cheti tofauti na ilivyokuwa kwa Kiingereza.

Matamko hayo ya serikali yalikuwa na athari si haba kwa lugha ya Kiswahili. Kiswahili kilibadilika kutoka kuwa lugha ya utawala wa kikoloni hadi kuwa lugha ya ukombozi, utambulisho na itikadi. Hata hivyo, mahusiano ya kitabaka yanayoakisiwa kiisimujamii hayakuweza kukifanya Kiswahili kiwe na nguvu na kikubalike zaidi miongoni mwa Watanzania ikilinganishwa na lugha ya Kiingereza ambayo kwa sasa imekita mizizi zaidi. Sababu iko wazi kwamba elimu kwa Watanzania wengi ina maana ya kujua Kiingereza na Kiingereza kinachukuliwa kuwa ndiyo elimu yenyewe. Ingawa mtazamo huo ni potofu, bado mpaka leo una mashiko miongoni mwa Watanzania wakiwemo wasomi waliobobea. Kwa mtazamo wa nadharia iliyoelezwa hapo awali, wanaungana na tabaka la watu wenye nguvu za kiuchumi na kwa pamoja kulinda maslahi yao kwa mwavuli wa lugha ya Kiingereza ambayo wanyonge wengi hawaijui.

Kwa mujibu wa Tibategeza (2009) kutumia Kiswahili lugha inayojulikana na wengi kulipunguza ujinga kutoka $75 \%$ hadi $17 \%$. Mpango wa Maendeleo wa Miaka Mitano 1969/1970 hadi 1973/1974 ulitarajiwa kwamba ifikapo 1971 Kiswahili kingechukua jukumu katika nafasi zote ambazo Kiingereza kilikuwa kinatumika. Hata hivyo, malengo hayo hayakutekelezwa na Kiingereza kinaendelea kutumika kwa sababu sawa na zile zinazotajwa na Galabawa na Senkoro (2006) kwamba kuna imani potofu kuwa kutumia Kiswahili kutashusha ubora wa elimu, kubadilisha lugha kunaambatana na gharama kubwa pamoja na 


\section{Mkwawa Journal of Education and Development, Vol. 1, Issue 1, 2017 DOI: https://doi.org/10.37759/mjed.2017.1.1.6}

hofu ya kutengwa na jamii ya kimataifa. Hata hivyo, ukweli ni kwamba hoja hzio zote zinatumika kama njia ya kuwatishia wale wanaotaka Kiswahili kitumike katika elimu. Jambo muhimu katika elimu ni kukomboa fikra za jamii husika kabla ya kufikiria nafasi ya kimataifa. Ukombozi huo hauwezekani kwa matumizi ya lugha ya kigeni katika elimu.

Tume ya Rais ya Mapitio ya Mfumo wa Elimu Nchini ya mwaka 1980, maarufu kama Tume ya Makweta, iliamua kuchunguza kipengele cha lugha ya kufundishia ingawa haikuwa mojawapo ya hadidu zake za rejea. Ripoti yake ya mwaka 1982 ilipendekeza Kiswahili kitumike kama lugha ya kufundishia katika elimu ya sekondari ifikapo mwaka 1985 na katika elimu ya juu mapema mara baada ya wahitimu wa kwanza wa mtaala wa Kiswahili. Hata hivyo, mpango huo ulifutwa mwaka 1983. Ingawa halikutolewa tamko hadharani, sababu ya uamuizi huo iko wazi kwamba ni athari za uhusiano wa Tanzania na Uingereza na nchi zingine za kibepari zinazonufaika na lugha ya Kiingereza, uliojengwa katika misingi ya ubwana na utwana na Kiingereza ndiyo lugha ya upatanisho baina ya kitovu cha kitovuni na kitovu cha pembezoni. Kwa hiyo kikataa lugha hiyo ni kukataa faida zinazotarajiwa hata kama ni kwa gharama ya kuua vipaji vya taifa nzima.

Wakati mapendekezo hayo yakipuuzwa, serikali ikakubali mapendekezo ya maofisa kutoka Baraza la Uingereza, Cripa na Dodd (1984) ya kutaka Kiingereza kifundishwe kwenye umri mdogo. Ushauri huo ulikuwa unakinzana na matokeo ya msingi ya utafiti wa maofisa hao wa Uingereza. Matokeo yao ya msingi yalionesha kwamba ni $10 \%$ tu ya wanafunzi wa kidato cha nne kwa wakati huo walikuwa na umilisi wa lugha ya Kiingereza kwa kiasi cha kufikiria kwamba ujifunzaji kwa Kiingereza ungeanza. Pendekezo lenye maslahi kwa Watanzania lingekuwa ni kuunga mkono pendekezo la Tume ya Makweta la kutaka Kiswahili kitumike kufundishia katika ngazi zote. Kwa kuwa Kiingereza ni lugha ya wenye nguvu na utashi wa kisiasa nchini kuhusu lugha ya Kiswahili uko chini, mambo yanaendelea kwa masilahi ya "bwana mkubwa" Uingereza na wateule wachache wa kwenye kitovu cha pembezoni na maswahiba wao.

\subsection{Suala la Lugha kwenye Sera Utamaduni, 1997}

Kwa mara ya kwanza suala la lugha Tanzania liliingizwa kwenye Sera ya Utamaduni (SU) mwaka 1997. Sera hiyo ilionesha nafasi ya lugha za asili kikiwemo Kiswahili na lugha za Kigeni. Kuhusu lugha ya kufundishia, SU katika Ibara ya 3.4 Ibara ndogo ya 3.4.1 na 3.4.2 inaweka bayana kwamba:

\subsubsection{Mpango maalumu wa kuiwezesha elimu na mafunzo katika ngazi zote} kutolewa katika lugha ya Kiswahili utaandaliwa na kutekelezwa.

3.4.2 Somo la Kiswahili litakuwa ni somo la lazima katika ngazi ya elimu ya awali, msingi na sekondari, na litahimizwa katika elimu ya juu. Aidha, ufundishaji wake utaboreshwa.

Hata hivyo Ibara ya 3.4.1 hapo juu haikutekelezwa licha ya kwamba lilikuwa ni tamko la serikali. Tangu wakati huo Kiswahili kimeendelea kuwa lugha ya kufundishia katika elimu ya awali, elimu ya msingi, elimu ya ualimu ngazi ya cheti (kwa sasa Stashahada ya Kawaida) na katika vyuo vya ufundi pamoja na mahakama za mwanzo. Kiingereza 
kimeendelea kuwa lugha ya kufundishia katika elimu ya sekondari elimu ya juu na kutumika kwenye mahakama za hakimu mfawidhi na mahakama za rufaa.

Inafaa kuweka bayana kuwa baadhi ya shule nchini zimekuwa zikifundisha kwa lugha ya Kiingereza katika ngazi ya elimu ya awali na elimu ya msingi kabla ya SEM, 2014. Shule hizo baadhi ni za binafsi na baadhi ni za serikali. Kama ilivyoelezwa mapema kwamba msimamo wa kisera ulitaka elimu ya msingi itolewe kwa lugha ya Kiswahili. Kutumia Kiingereza kufundishia elimu ya msingi bila tamko la kisera inawezekana kwa sababu Kiingereza ni lugha ya tabaka tawala, lugha ya wasomi, lugha ya matajiri, ima kwa kauli moja lugha ya tabaka lenye nguvu. Tutarejea kwenye kaifa hii hapo baadaye hasa kuhusu athari za uamuzi huo.

\subsection{Suala la Lugha kwenye Sera ya Elimu na Mafunzo ya 1995}

Sera ya Elimu na Mafunzo ya mwaka 1995 (kwa hiyo, SEM, 1995) pamoja na maboresho ya uanzishwaji na umiliki wa shule kutoka kumilikiwa na serikali pekee hadi umiliki wa serikali na binafsi, tamko kuhusu lugha ya kufundishia linafuata tamko la kuweka mpango wa elimu katika ngazi zote kutolewa katika lugha ya Kiswahili la SU, 1997. SEM, 1995 inakipangia Kiswahili kutumika katika ngazi za chini za elimu. Katika Ibara ya 5.2.3 na 5.3.7 inaweka bayana kwamba:

\subsubsection{Lugha ya kufundishia elimu ya awali itakuwa Kiswahili na Kiingereza} litakuwa somo la lazima.

\subsubsection{Lugha ya kufundishia elimu ya msingi itakuwa Kiswahili na Kiingereza} litakuwa somo la lazima (Tafsiri yangu)

Wakati Kiswahili kinapewa nafasi ya kuwa lugha ya kufundishia katika ngazi hizo za chini za elimu, Kiingereza katika nyakati za sera zote mbili kimekuwa kikitumika kama lugha ya kufundishia elimu ya sekondari na elimu ya juu. Sababu za kufanya hivyo ziko wazi, kwanza ni sababu za kihistoria ambazo zimeibua na kuimarisha Ubeberu wa Kiisimu uliopata mashiko kutokana na kasumba ya kutawaliwa. Dhana ya kasumba hapa inatumika sawa na ile inayofasilliwa na TUKI (2013) kuwa ni "mabadiliko ya fikra za mtu yanayosababishwa na elimu au utamaduni wa jamii, yanayomfanya afuate mwenendo usio muafaka na utamaduni wake au utu wake wa asili" kwa upande mwingine dhana ya Ubeberu wa Kiisimu imetumika sawa na ile ya Phillipson (1997) kuwa ni mwega wa kinadharia uliobuniwa kufafanua mpangilio msonge wa kiisimu na mwenendo wa baadhi ya lugha hutumika zaidi kuliko zingine. Inahusisha pia miundo na itikadi zinazochochea mpangilio huo na kubainisha dhima ya wataalamu wa isimu na lugha katika kuendeleza mkondo huo wa fikra. Fasili hii inaakisi muktadha wa uhusiano wa Kiingereza na Kiswahili ambapo Kiingereza kinatumika katika maeneo mengi na nyeti zaidi kuliko Kiswahili na mifumo ya mamlaka na tabaka la wasomi wakichukulia hali hiyo kama ukweli usiosailika.

Hadhi ya lugha ni suala la kijamii kwa sababu jamii ndiyo inayoamua lugha ipi iwe na hadhi zaidi kuliko nyingine. Kama ilivyoelezwa awali sera yoyote ni zana ya kuhalalisha matakwa ya tabaka lenye nguvu. Sera katika muktadha wa makala haya imetumika kutukuza lugha ya Kiingereza na kutweza lugha ya Kiswahili. Kuweka sera kuhusu jambo fulani ni kuongeza dhamira ya kisiasa kwenye jambo husika ingawa mara nyingi nia thabiti inapokosekana sera 
nzuri hubakia kuwa mapambo kwenye mashubaka ya waliozitunga. Kama ilivyoelezwa awali kwamba kuna shule zilizoanzishwa zikiwa zinatumia lugha ya Kiingereza kufundishia katika ngazi ya elimu, ambapo kwa mujibu wa sera, Kiswahili kilipaswa kutumika. Shule hizo baadhi ni za mashirika, watu binafsi na baadhi ni za serikali, serikali ambayo kimsingi inapaswa kusimamia utekelezaji wa sera. Kuanzishwa kwa shule hizo na kukubaliwa na serikali bila tamko rasimi la kisera kunaleta fasili mbili:

Fasili ya kwanza ni kukosa utashi wa kisiasa wa kukuza Kiswahili ili kitumike kama lugha ya kufundishia na kwamba serikali iliridhishwa na matumizi ya lugha ya Kiingereza licha ya kuwapo kwa tafiti nyingi zinazoweka bayana kwamba kushuka kwa kiwango cha elimu pamoja na mambo mengine kunachangiwa na tatizo la lugha (Qorro, 2007). Tunasema ni kukosa utashi wa kisiasa kwa sababu suala hapa sio la wananchi kutaka au kuto kutaka lugha gani itumike katika elimu. Muhimu hapa ni kwamba serikali ina wajibu wa kuweka mipango yenye tija katika elimu itakayowanufaisha watu wote bila kujali tofauti zao za kiuchumi. Kutoa kipaumbele kwenye lugha ya Kiingereza ni kunufaisha kundi dogo kabisa kama ilivyodokezwa kuwa ni chini ya asilimia tano ya Watanzania wote. Katika hali kama hii ni vigumu kufikiria kwamba hii sio 'hujuma'. Fasili ya pili ni kwamba serikali ilitumia nafasi hiyo ili kujiridhisha ikiwa Kiingereza kinafaa kuwa lugha ya kufundishia katika ngazi ya elimu ya msingi na awali ama la. Kwa maneno mengine, serikali ilifanya utafiti wake ili hatimaye iweze kufanya maamuzi makubwa ya kisera na ambayo yanaweza kufunga mjadala wa lugha ipi iwe ya kufundishia katika ngazi zote za elimu. Kuzinduliwa kwa SEM, 2014 ni hitimisho na majibu ya utafiti wake pamoja na hitimisho la mjadala uliodumu kwa takribani miongo mitatu kuhusu lugha ipi iwe ya kufundishia katika ngazi zote za elimu. Swali la kujiuliza hapa Kiswahili kina nafasi gani kwenye SEM, 2014 na nini fasili ya nafasi hiyo katika muktadha wa uhusiano wake na lugha ya Kiingereza? Kunaweza kuwa na fasiri kwamba serikali imetoa uhuru ili kila mzazi achague, kwa maoni ya makala haya huko kutakuwa ni kutoa hitimisho rahisi na kukimbia wajibu. Hii inatokana na ukweli kwamba tafiti zilizopo (Qorro, 2006) zinaonesha wazi kwamba kutumia lugha za asili kufundishia kuna manufaa zaidi kuliko kutumia lugha za kigeni. Hata hivyo katika hatua hii ni jambo la welekea kuchunguza suala la lugha ya kufundishia kwenye SEM, 2014.

\subsection{Suala la Lugha kwenye Sera ya Elimu na Mafunzo, 2014}

Licha ya tamko la SEM, 2014 lenye kuashiria ahueni kwa lugha ya Kiswahili, kimsingi, SEM, 2014 ina mwelekeo wa kudhohofidha Kiswahili kwenye mfumo wa elimu kama lugha ya kufundishia na Kiingereza kuchukua dhima hiyo katika ngazi zote. Sera hiyo inazua shaka kubwa ya kuwepo kwa utashi wa dhati wa kukiinua Kiswahili. Kuna ukinzani wa kimaudhui baina ya SEM, 2014 na hali halisi ya kiisimujamii. Kama itakavyooneshwa baadaye, hadhi inayopewa Kiswahili kwenye SEM, 2014, hasa unapozingatia uhusiano wa Kiswahili na Kiingereza kiisimujamii, haiakisi hali halisi. Hili linatokana na matamko yaliyopo kwenye SEM, 2014 katika sura ya tatu Ibara ya 3.2.19 na 3.2.20 kama ifuatavyo:

3.2.19. Lugha ya Taifa ya Kiswahili itatumika kufundishia na kujifunzia katika ngazi zote za elimu na mafunzo na Serikali itaweka utaratibu wa kuwezesha matumizi ya lugha hii kuwa endelevu na yenye ufanisi katika kuwapatia walengwa elimu na mafunzo yenye tija kitaifa na kimataifa. 
3.2.20. Serikali itaendelea na utaratibu wa kuimarisha matumizi ya lugha ya Kiingereza katika kufundishia na kujifunzia katika ngazi zote za elimu na mafunzo.

Tamko la SEM, 2014 Ibara ya 3.2.19 kimaudhui linafufua matumaini ya SEM, 1997 katika Ibara ya 3.4.1 ambalo halikutekelezwa. SEM, 2014 inakipa Kiswahili na Kiingereza hadhi sawa katika elimu. Kimsingi usawa huo kiisimujamii unazua changamoto kwa sababu mwelekeo wa jamii kuhusu lugha na elimu ni chanya zaidi kwenye Kiingereza kuliko kwenye Kiswahili. Suala la elimu kunasibishwa zaidi na lugha za kigeni linaelekea kuwa la kawaida katika nchi nyingi za Afrika zilizokuwa zimetawaliwa na mataifa ya kibeberu (Momanyi, 2007). Katika hali hiyo, tamko la 3.2.19 linakinzana na lile la 3.2.20. Uwiano wa matamko hayo haupo. Wakati Kiswahili kitaanza kutumika (miaka mitatu sasa mpango haujawekwa) Kiingereza kitaimairishwa! Hali hii ni kuendelea kufisha nafasi ya Kiswahili kwenye mfumo wa elimu na Kiingereza kuendelea kuimarika kwa sababu hadhi ya lugha hizo kiisimujamii sio sawa. Kiingereza ni lugha inayohusishwa na maslahi ya kijamii kama kazi nzuri, mawasiliano ya kimataifa, sayansi na teknolojia kwa ujumla jamii inaona kuwa ukwasi na maisha bora vina ukuruba mkuruba mkubwa na lugha ya Kiingereza. Hii ndio sababu makala haya yanatoa hoja kwamba lugha ya Kiswahili na lugha ya Kiingereza visiingie kwenye mfumo wa elimu katika sura ya ushindani kwa kuwa Kiingereza kimeshashinda kabla ya SEM, 2014. Kiswahili kinapaswa kupewa rasilimali na utashi maalumu ili kitekeleze jukumu lake la ukombozi wa jamii pana.

Ingawa haijaelezwa jinsi utekelezaji wake utakavyokuwa na pamoja na ukweli kwamba kuna modeli mbalimbali, namna moja kati ya mbili zinaweza kutumika. Kwanza ni kuwa na mitaala inayofundishwa kwa Kiswahili kuanzia elimu ya awali hadi elimu ya juu kwa masomo yote kwa upande mmoja na mitaala inayofundishwa kwa Kiingereza kuanzia elimu ya awali hadi elimu ya juu kwa uapande mwingine. Ikiwa modeli hii itatumika na mambo kutokea kama inavyohitajika kwenye mfumo wa elimu wenye lugha uwili itakuwa ni hatua muhimu kwa lugha ya Kiswahili.

Hata hivyo, changamoto iliyopo ni jinsi ya kutengeneza mizania baina ya aina hizo mbili za elimu kwa lugha tofauti. Kiingereza tayari kinaaminiwa na jamii kwamba kina uwezo wa kutoa elimu bora. Jamii haiamini kama Kiswahili kinaweza hasa linapokuja suala la elimu ya sekondari na elimu ya juu. Kutokana na hali hiyo ni mantiki kufikiria kwamba watu wengi wataamua kujiunga shule za mtaala wa Kiingereza ili kupata elimu bora ingawa tatizo la kipato ni changamoto nyingine inayoweza kuwarudisha kwa shingo upande katika shule za mitaala ya Kiswahili. Kutokana na kasumba iliyopo ni vigumu mtu binafsi kuanzisha shule ya sekondari na kutumia mtalaa Kiswahili kama lugha ya kufundishia. Kutokana na hali hiyo ni wazi kwamba taasisi nyingi za elimu na mafunzo za mitalaa ya Kiswahili zitaendelea kuwa za serikali.

Shule nyingi hasa za msingi na sekondari miundombinu yake ni mibovu na wanaosoma kwenye shule hizo wanahusishwa na umaskini na kwa hiyo na elimu duni. Ni dhahiri kwamba Kiswahili kitaendelea kunasibishwa na elimu duni na umasikini wakati Kiingereza kitaendelea kunasibishwa na elimu bora na ukwasi. Katika mazingira haya kuchagua Kiingereza sio suala la kukukipenda bali kimejengewa mazingira ya kuhusishwa na mafanikio ya kimaisha katika maeneo yote yanayomzunguka binadamu jambo ambalo kwa mujibu wa Phillipson (1997) ni matokeo ya athari za ubeberu wa kiisimu. Jitihada za 
watanzania zitakuwa kupata elimu bora ambayo kwa fasili ya kwanza ni lugha ya Kiingereza. Hatimaye watu watalazimika kutafuta fedha kugharimia elimu kwa Kiingereza na mwisho wa siku Kiswahili kitaondolewa kwenye mfumo wa elimu kwa madai kuwa wanajamii wenyewe wamechagua kufundishwa kwa lugha ya Kiingereza. Hii inakinzana na hali halisi kwa sababu mazingira hayaoneshi uhuru wa kuchagua bali mifumo imejengwa jamii kufanya kulingana na matakwa yake.

Kama hilo lisipowezekana watabakia kwenye shule zinazofundishwa kwa Kiswahili kama ilivyoelezwa awali kwa shingo upande kwa sababu mafanikio ya kijamii hayaelekei kufungamana na mfumo wa elimu ambao gharama zake wanazimudu. Mfumo unaotumia lugha ya Kiswahili. Kimsingi kitakachotokea sio uchaguzi bali nguvu za kisiasa na kiuchumi hazitoi nafasi kwa mtu duni kuchagua. Tabaka la wanyonge ambao ni wengi wataendelea kutafuta jahala yao kwa kadiri tabaka la wenye nguvu linavyowaonea huruma. Ni dhahiri kuwa tamko katika SEM, 2014 kuhusu lugha ya kufundishia ni lenye kutoa matumaini kwa lugha ya Kiswahili lakini uzoefu wa utekelezaji wa matamko kama katika sera zilizotangulia na kwa kuzingatia hali ya kiisimujamii, tamko hilo ama litekelezwe kwa tahadhari ya dhati ya kukiendeleza Kiswahili au vinginevyo halina nia njema kwa mustakabali wa lugha ya Kiswahili kama lugha ya kufundishia kwa sababu nguvu za ushindani kwa lugha ya Kiswahili dhidi ya lugha ya Kiingereza ni ndogo kama inavyobainika baina ya jamiilugha zinazotumia lugha hizo.

Namna ya pili ni kutumia modeli ya 50\%-50\% iliyopendekezwa na Tibategeza (2009). Modeli hiyo inahitajia kwamba kama kuna lugha mbili za kufundishia, kwa mfano Kiswahili na Kiingereza kwa Tanzania, kila lugha itumike kufundisha idadi sawa ya masomo katika ngazi ya elimu ya awali hadi elimu ya sekondari. Kusema ukweli ikitumika modeli hii bado utakuwa ni mwanzo mzuri kwa lugha ya Kiswahili ikilinganishwa na hali ilivyo hivi sasa. Hii inatokana na ukweli kwamba kutoka kutotumika kabisa sekondari hadi kutumika kwa asilimia hamsini ni hatua ya maendeleo. Hata hivyo modeli hii ina matatizo makubwa mawili. Kwanza, kama hilo litatekelezwa haizungumzii lolote kuhusu ufundishaji wa kiwango cha vyuo vya kawaida, kati na vya elimu ya juu. Kutokana na hali ilivyo ni mantiki isiyowezekana kufikiria kwamba elimu ya juu itafundishwa kwa Kiswahili bali kinyume chake inawezekana. Ikiwa hali hiyo ni sawa basi mwitikio wa jamii utakuwa ni kujinasibisha na lugha ya elimu ambayo inatumika ngazi za chini na inatumika ngazi ya juu. Mazingira hayo ndiyo yanayotishia nafasi ya Kiswahili kwenye mfumo wa elimu.

Tatizo la pili linahusu mgawanyo wa masomo ya kufundishwa kwa Kiswahili na yale ya kufundishwa kwa Kiingereza, masomo yote ya sayansi yanapendekezwa yafundishwe kwa Kiingereza isipokuwa hisabati kwa elimu ya msingi. Mgawanyo huo unaendeleza kasumba ya muda mrefu kwamba lugha ya Kiswahili bado ni changa kwa ajili ya kufundishia masomo ya sayansi na teknolojia. Hali hiyo inafisha dhana ya 50\% - 50\%. Mtazamo huo ni wa hatari kwa sababu chachu ya lugha kukua ni kuipa majukumu na si kuisubiri ikue kwanza kama mmea unaohitajika kwa ajili ya ujenzi wa nyumba baadaye. Kwa hiyo, modeli hiyo bado ni tishio kwa mustakabali wa lugha ya Kiswahili hasa kutokana na uhusiano wake na lugha ya Kiingereza kwa mtazamo wa kiisimujamii.

\subsection{Hitimisho}


Kinachotokea kwenye SEM, 2014 ni mwelekeo kinzani wa maudhui yake na hali halisi ya kiisimujamii dhidi ya mustakabali wa lugha ya Kiswahili kwenye mfumo wa elimu nchini. Sera ya Elimu na Mafunzo ambayo ndani yake kuna sera ya lugha kuna matamko mawili ambayo yanakinzana baina yake na pia na hali halisi ya kiisimujamii. SEM, 2014 inataka uwili lugha katika mfumo wa elimu. Hiyo ni hatua nzuri kwa lugha ya Kiswahili ikiwa utekelezaji wake utazingatia tofauti za nguvu zilizopo baina ya lugha ya Kiswahili na lugha ya Kiingereza. Kuacha sura ya ushindani kwamba kila lugha itumie nguvu na rasilimali zake bila kujenga mazingira ya Kiswahili kukua itaendelea kujenga mustakabali dhaifu wa lugha ya Kiswahili. Matokeo ya mwisho ya mustakabali huo dhaifu ni kudidimia kwa lugha ya Kiswahili kwa sababu katika utendaji wa kila siku sera ina nafasi muhimu katika utashi wa kisiasa. Matokeo ya mwisho sio tu Kiswahili kutoweka kwenye mfumo wa elimu vilevile itaendelea kusababisha kile anachokiita Senkoro (2007) "mauaji ya halaiki" ya akili za watoto wa Tanzania kwa kulazimishwa kujifunza kwa lugha ya kigeni. Matamko mengi yamewahi kutolewa bila kutekelezwa hata hivyo hatuwezi kuchukulia kwamba hali ya mambo itakuwa namna hiyo ndio kwa maana tunatoa wito wa tahadhali katika utekelezaji wa SEM, 2014. Kutekeleza sera hiyo bila upendeleo maalumu kwa lugha ya Kiswahili matokeo yake yanaweza kuwa mabaya zaidi. Kimsingi tunahitaji utashi wa dhati wa kunusuru vipaji vya watoto. Wito wa makala haya ni kwamba pendekezo la Tume ya Makweta linapaswa kuhuishwa wakati huu sambamba na SEM, 2014 kama kipindi cha mpito kuelekea matumizi ya lugha ya Kiswahili pekee katika ngazi zote za elimu. Madai ya Senkoro (2005) kwamba ni vigumu kuinua ubora wa elimu bila kuzingatia kigezo cha lugha ya kufundishia lazima yazingatiwe kikamilifu na watunga sera kwa faida ya wengi ili kuhuisha malengo ya elimu.

\section{Marejeleo}

BAKITA (2004). Tafsiri sanifu (Standard Translation). Dar es Salaam: BAKITA.

Boroditsky, L. (2001). Does Language Shape Thought?: Mandarine \& English Speakers' Conception of Time. Cognitive Psychology, (43). 1-22.

Criper, C. \& Dodd, B. (1984). Report on the Teaching of English Language and its use as a Medium of Education in Tanzania. Dar es Salaam: The British Council.

Fasold, R. (1990). The Social Linguistics of Language, Oxford: Basil Blackwell.

Galabawa, J. C. J. \& Senkoro, F. E. M. K. (2006). Implications of Changing the Language of Instruction in Secondary and Tertiary Eduction in Tanzania. In Brock-Utne, B., Desai, Z. \& Qorro, M. (Wah). Focus on Fresh Data on the Language of Instruction Debate in Tanzania and South Africa. African Minds: 41-58.

Krashen, S. (1981). Second Language Acquisition and Second Language Learning, California: Pergamon Press.

Malekela, G. A. (2006). Performance in the Primary School Leaving Examination (PSLE): A comparison Between Kiswahili and English in Focus on Fresh Data on the Language of Instruction Debate in Tanzania and South Africa. ktk Brock-Utne, B., Zubaida D. and Qorro, M. (Wah.) Cape Town: African Minds: 85-92.

Mekacha, R. D. K. (1995). Kwanini bado Kiingereza ni lugha ya kufundishia Elimu ya Juu Tanzania, katika Kioo cha Lugha, Juz. 2. 28-46.

Mekacha, R. D. K. (2011). Isimujamii: Nadharia na Muktadha wa Kiswahili, Dar es Salaam: TUKI. 
Momanyi, C. (2007). Suala la Mielekeo ya Lugha, katika Senkoro, F.E.M.K (Mh) Lugha na Fasihi ya Kiswahili Afrika Mashariki, Dar es Salaam: CHAKAMA.

Mukangara, D. R. (2001). Forms and Reforms of Constitution - Making with Reference to Tanzania, katika UTAFITI [Mfululizo Mpya] Special Issue, Vol. 4. 19998-2001: 131-150.

Mulokozi, M. M. (2002). Kiswahili as a National and International Language. Dar es Salaam: IKS.

Phillipson, R. (1997). Realities and Myths of Linguistic Imperialism. Journal of Multilingual and_Multicultural Development, 18:3, 238-248.

Qorro, M. (2007). Matatizo ya Kufundisha kwa Lugha za Kigeni katika nchi za Afrika: Kwa mfano Kiingereza katika shule za Sekondari Tanzania, ktk Senkoro, F.E.M.K (Mh.) Lugha na Fasihi ya Kiswahili Afrika Mashariki. Dar es Salaam: CHAKAMA.

Rogers, C. (1969). Freedom to Learn: A View of What Education Might Become, $1^{\text {st }}$ Edition. London: Routledge Publishers.

Rubagumya, C. M. (1989). English-Medium Instruction in Tanzania Secondary Schools: A Conflict of Aspirations and Achievements. Jornal of Multlingual and Multcultural Development Juz. 11: 107-115.

Rubanza, Y. I. (1996). Can a Three-Tier Language Policy Work in Tanzania? A new Perspective, A Paper Presented at the UCLA African Studies Centre". Dar es Salaam: Noon Seminar Series.

Senkoro, F. E. M. K. (2005). Language of Instruction: The Forgotten Factor in Education Quality and Standards in Africa". Dar es Salaam: University of Dar es Salaam.

Senkoro, F. E. M. K. (2007). Mauaji ya Halaiki ya watoto wa Tanzania kupitia Lugha ya Kufundishia, katika Senkoro, F.E.M.K (Mh) Lugha na Fasihi ya Kiswahili Afrika Mashariki. Dar es Salaam: CHAKAMA.

Tempels, P. (1959). Bantu Philosophy. London: University of London, Institute of Education

Tibategeza, E. R. (2009). Language in Education Planning in Tanzania: A Sociolinguistic Analysis. Tasinifu ya Uzamivu. (haijachapishwa) University of Free State.

TUKI (2013). Kamusi ya Kiswahili Sanifu, Dar es Salaam: TUKI \& OXFORD University Press.

URT (1995). Education and Training Policy. Dar es Salaam: Government Printing Press.

Wizara ya Elimu na Mafunzo (2014). Sera ya Elimu na Mafunzo. Dar es Salaam: Mpiga chapa wa Serikali.

Wizara ya Elimu na Utamaduni (1997). Sera ya Utamaduni. Dar es Salaam: Mpiga chapa wa Serikali. 